\title{
The atmospheric structure and fundamental parameters of the red supergiants AH Sco, UY Sct, and KW Sgr
}

\author{
B. Arroyo-Torres ${ }^{1}$, M. Wittkowski ${ }^{2}$, J. M. Marcaide ${ }^{1}$, and P. H. Hauschildt ${ }^{3}$
}

\author{
${ }^{1}$ Dpt. Astronomia i Astrofísica, Universitat de València, C/Dr. Moliner 50, 46100, Burjassot, Spain e-mail: belen. arroyo@uv . es \\ 2 ESO, Karl-Schwarzschild-St. 2, 85748, Garching bei München, Germany \\ ${ }^{3}$ Hamburger Sternwarte, Gojenbergsweg 112, 21029, Hamburg, Germany
}

Received 14 December 2012 ; accepted 18 April 2013

\section{ABSTRACT}

\begin{abstract}
Aims. We present the atmospheric structure and the fundamental properties of the red supergiants (RSGs) AH Sco, UY Sct, and KW Sgr based on VLTI/AMBER observations.

Methods. We carried out spectro-interferometric observations of AH Sco, UY Sct, and KW Sgr in the near-infrared K band (1.92$2.47 \mu \mathrm{m})$ with the VLTI/AMBER instrument with spatial and spectral resolutions of 3 milliarcseconds and 1500, respectively, and compared the data to a new grid of hydrostatic PHOENIX model atmospheres.

Results. In our visibility data, we observe molecular layers of water and CO in extended atmospheres. For a uniform disk modeling, we observe size increases at the water band centered at $1.9 \mu \mathrm{m}$ of $10 \%$ to $25 \%$ and at the CO bandheads at $2.3-2.5 \mu \mathrm{m}$ of $20 \%-35 \%$ with respect to the near-continuum bandpass at around $2.20 \mu \mathrm{m}$. Our near-infrared spectra of AH Sco, UY Sct, and KW Sgr are well reproduced by the PHOENIX model atmospheres. The continuum visibility values are consistent with a limb-darkened disk as predicted by the PHOENIX models. However, the model visibilities do not predict the large observed extensions of the molecular layers. Comparing the continuum visibility values to PHOENIX models, we estimate the Rosseland-mean photospheric angular diameters of AH Sco, UY Sct, and KW Sgr to be 5.81 \pm 0.15 mas, 5.48 \pm 0.10 mas, and 3.91 \pm 0.25 mas, respectively. Together with the distance and the spectro-photometry, we calculate radii of $1411 \pm 124 \mathrm{R}_{\odot}$ for AH Sco, $1708 \pm 192 \mathrm{R}_{\odot}$ for UY Sct, and $1009 \pm 142 \mathrm{R}_{\odot}$ for KW Sgr and effective temperatures of $3682 \pm 190 \mathrm{~K}$ for AH Sco, $3365 \pm 134 \mathrm{~K}$ for UY Sct, and $3720 \pm 183 \mathrm{~K}$ for KW Sgr.

Conclusions. AH Sco, UY Sct, and KW Sgr exhibit extended atmospheric layers of $\mathrm{H}_{2} \mathrm{O}$ and CO. The PHOENIX atmosphere models predict the spectra and the continuum visibility values, but cannot reproduce the large extensions of the molecular layers. This indicates that the opacities of the molecular bands are included, but that the model atmospheres are too compact compared to the observations. The observed extended layers may be levitated by processes such as pulsation or convection, which are not included in the hydrostatic atmospheric models. The location of the targets in the HR-diagram is confirmed to be close to, and possibly slightly to the right of, the Hayashi limit of recent evolutionary tracks corresponding to masses between about $20 \mathrm{M}_{\odot}$ and $40 \mathrm{M}_{\odot}$.
\end{abstract}

Key words. supergiants - Star: fundamental parameters - Star: atmospheres - Hertzsprung-Russell and C-M diagrams - Star: individual: AH Sco, UY Sct and KW Sgr.

\section{Introduction}

Our research aims to investigate the circumstellar environment of red supergiants (RSGs) close to the photosphere, to obtain fundamental parameters, and to locate the stars in the Hertzsprung-Russell (HR) diagram. The characterization and location in the HR diagram are highly relevant to calibrating stellar evolutionary models for massive stars and understanding their further evolution towards Wolf-Rayet (WR) stars or supernovae.

The fundamental parameters and the atmospheric structure of RSGs have not been characterized well yet. Recent spectrophotometric observations compared to MARCS model atmospheres caused a dramatic revision of the location of RSGs in the HR diagram (Levesque et al.2005, Massey et al.2006). An initial interferometric survey of RSGs of spectral types G8-M2 (van Belle et al. 2009) is in broad agreement with these new spectroscopic results, with the exception of K type RSGs.

Interferometric observations of RSGs at continuum bandpasses have shown that the continuum photosphere can be well described by a limb-darkened disk (Perrin et al.2004, Ohnaka et al. 2011) that is consistent with MARCS (Haubois et al. 2009) and PHOENIX (Wittkowski et al.2012) model atmosphere predictions. A study of the close red supergiant Betelgeuse (Ohnaka et al. 2009, 2011) suggests there are extended CO layers, which cannot be accounted for by classical hydrostatic model atmospheres. Other studies suggest the presence of extended and asymmetric water vapor layers in RSGs (Perrin et al.2004, 2005, Wittkowski et al. 2012). The mechanisms that levitate the mass to radii where the wind can be accelerated by the radiation pressure on dust grains have not yet been understood for RSGs, but are crucial for the mass-loss process (e.g., Bladh \& Höfner 2012).

Recently, we used the VLTI/AMBER instrument to study the characteristics of the molecular layers of Mira variables and the red supergiant VY CMa, and demonstrated that this instrument, with its spectro-interferometric capabilities, is well suited to revealing both the angular diameter of the continuum photosphere at bandpasses with little contamination by molecular bands and the structure of the extended atmosphere (Wittkowski et al.|2011, 2012). In this paper we extend VLTI/AMBER studies to a larger sample of RSGs covering spectral types M1-M5, extending the sample of van Belle et al. (2009) to cooler spectral types.

The remainder of this paper is structured as follows. In Sect. 2 we describe our AMBER observations, the data reduction, and the first results. In Sect. 3 we explain the atmospheric model 
Table 1. VLTI/AMBER observations

\begin{tabular}{lcccc}
\hline \hline $\begin{array}{l}\text { Target } \\
\text { (Sp. type) }\end{array}$ & Date & $\begin{array}{c}\text { Mode } \\
\text { K- } \\
\mu \mathrm{m}\end{array}$ & $\begin{array}{c}\text { Baselines } \\
\text { BL1/BL2/BL3 } \\
\mathrm{m}\end{array}$ & PA \\
\hline AH Sco & $2010-06-04$ & 2.1 & $40.6 / 40.4 / 62.3$ & $168 /-51 /-91$ \\
(M4-5) & $2010-06-04$ & 2.3 & $40.8 / 45.2 / 66.7$ & $159 /-63 /-99$ \\
UY Sct & $2010-06-04$ & 2.1 & $37.6 / 43.4 / 58.5$ & $163 /-64 /-103$ \\
(M4) & $2010-06-04$ & 2.3 & $36.9 / 37.4 / 49.8$ & $172 /-56 /-102$ \\
KW Sgr & $2012-04-21$ & 2.1 & $62.9 / 71.5 / 70.1$ & $64 /-178 / 129$ \\
(M1.5) & $2012-04-21$ & 2.3 & $63.7 / 71.4 / 71.5$ & $73 /-170 / 137$ \\
\hline
\end{tabular}

Notes. The AMBER instrument mode is K-2.1 $\mu \mathrm{m}$ (medium-resolution mode with range 1.92-2.26 $\mu \mathrm{m})$ or $\mathrm{K}-2.3 \mu \mathrm{m}(2.12-2.47 \mu \mathrm{m})$. The baseline is the projected baseline length for the AT VLTI baseline used.

used. In Sect. 4 we report the results obtained from the model fitting and estimate the fundamental parameters. In Sect. 5 . we discuss the atmospheric structure of our targets. Finally, in Sect. 6 we summarize our conclusions.

\section{Observations, data reduction, and results}

We observed the RSGs AH Sco (Simbad spectral type M45 Iab), UY Sct (M4 Ia), and KW Sgr (M1.5 Iab) with the ESO Very Large Telescope Interferometer (VLTI), utilizing three of the Auxiliary Telescopes of $1.8 \mathrm{~m}$ diameter. We used the Astronomical Multi-BEam combineR (AMBER) with the external fringe tracker FINITO (Petrov et al. 2007). We worked in medium-resolution mode $(\mathrm{R} \sim 1500)$ in the $\mathrm{K}-2.1 \mu \mathrm{m}$ and $\mathrm{K}$ $2.3 \mu \mathrm{m}$ bands (together covering wavelengths between $1.9 \mu \mathrm{m}$ and $2.5 \mu \mathrm{m})$. We made our observations as sequences of calsci-cal (sci is the target and cal the calibrator), observing five scans for each of them. The log of the observations is reported in Table 1 The integration time (DIT) of each frame was $500 \mathrm{~ms}$. The used calibrators were chosen from the ESO Calibrator Selector CalVin based on the catalog of Lafrasse et al. (2010). We chose $\tau$ Sgr (RA 1906 56.4, DEC -27 40 13, spectral type K1 III and angular diameter $3.83 \pm 0.04 \mathrm{mas}$ ) as an interferometric calibrator for AH Sco and UY Sct. For KW Sgr we chose HR 6583 (RA 1742 51.1, DEC -36 56 44, spectral type K5III and angular diameter $2.48 \pm 0.18 \mathrm{mas}$ ) and $11 \mathrm{Sgr}$ (RA 181143.3 , DEC -23 42 04, spectral type K0 III and angular diameter $1.43 \pm 0.10$ mas).

Raw visibility and closure phase values were obtained from our AMBER data using the latest version of the amdlib data reduction package (Tatulli et al. 2007; Chelli et al. 2009). We appended all scans taken consecutively from the same source. Later, we selected and averaged the resulting visibilities of each frame using different criteria. In our case, the criteria were based on the baseline flux and on the fringe signal-to-noise ratio (SNR, for more information see the AMBER Data Reduction Software User Manual 1 ). The baseline flux of baseline $\mathrm{i}-\mathrm{j}$ is defined as $\sqrt{f_{i} f_{j}}$, with $f_{i}$ and $f_{j}$ being the flux from stations $\mathrm{i}$ and $\mathrm{j}$ comprising the baseline $\mathrm{i}-\mathrm{j}$. We selected all frames that have a baseline flux that is, at least, three times larger than the associated noise and $80 \%$ of the remaining frames with best fringe SNR. After that, we performed the absolute wavelength calibration by correlating the AMBER flux spectra with a reference spectrum that included the AMBER transmission curves, the telluric spectrum estimated with ATRAN (Lord 1992), and the expected stellar spectrum, using the BS 4432 spectrum from Lançon \& Wood

\footnotetext{
${ }^{1}$ http://www.jmmc.fr/doc/approved/JMMC-MAN-2720-0001.pdf
}

(2000). We selected this star because it has a spectral type similar to our calibrators (the spectral type of our calibrator are: K1 III, K5 III, and K0 III and the spectral type of BS 4432 is K4.5 III). Then, we calibrated the flux and the visibility spectra. A relative flux calibration of the target was performed by using the calibrator and the BS 4432 spectrum. Calibrated visibility spectra were obtained by using the average of two transfer function measurements taken before and after each science target observation. For the observation of AH Sco with the K-2.3 setting, the calibrator scans taken before had a low quality. In this case we used only one calibrator. The error of the transfer function $(\Delta T)$ was calculated as a function of two terms $\left(\mathrm{T}_{A}\right.$ and $\left.\mathrm{T}_{B}\right)$. The first one was obtained by error propagation $\left(T_{A}=1 / V_{\text {inst }} * V_{c a l}^{m}\right)$, and the second one as $T_{B}=\left|T_{1}-T_{2}\right| / 2$, where $\mathrm{T}_{1}$ and $\mathrm{T}_{2}$ are the transfer functions of $\mathrm{cal}_{1}$ and $\mathrm{cal}_{2}$. When we used only one calibrator, we set $\mathrm{T}_{B}$ to a value of 0.05 , as obtained from our other observations.

Figures 1, 2, and 3 show the resulting flux and visibility data of AH Sco, UY Sct, and KW Sgr, respectively, together with predictions by model atmospheres, which will be discussed in the following sections.

The normalized flux spectra (top panels of Figs. 1, 3) show typical spectra of red supergiants, as observed for instance by Lançon et al. (2007). They show a decreasing flux between $1.9 \mu \mathrm{m}$ and $2.5 \mu \mathrm{m}$ and exhibit strong absorption features of CO. The flux variations at wavelengths below about $2.05 \mu \mathrm{m}$ are due to a higher noise level, possibly caused by the lower atmospheric transmission. These features are not seen in the visibility. The visibility curves (2nd panels) show a maximum near $2.25 \mu \mathrm{m}$, and a decrease towards the water bands (centered at $1.9 \mu \mathrm{m}$ ) and at the positions of the $\mathrm{CO}$ bandheads ( $2.3 \mu \mathrm{m}$ to $2.5 \mu \mathrm{m}$ ).

The uniform disk diameter calculated from our data as a function of wavelength (3rd panels), exhibits a minimum with a constant diameter at 2.20-2.25 $\mu \mathrm{m}$, and it increases in the $\mathrm{H}_{2} \mathrm{O}$ band and the $\mathrm{CO}$ bandheads. In the water band, the increase is about $25 \%$ for AH Sco, $16 \%$ for UY Sct, and $10 \%$ for KW Sgr compared to the near-continuum bandpass $(2.15-2.25 \mu \mathrm{m})$. In the $\mathrm{CO}$ bandheads it increases by about $65 \%$ for AH Sco, $45 \%$ for UY Sct, and $25 \%$ for KW Sgr. The decrease in the visibility and the corresponding increase in the UD diameter indicates a larger contribution from extended intensity at the molecular bands (water vapor and $\mathrm{CO}$ ). These results prove there are extended molecular layers of $\mathrm{H}_{2} \mathrm{O}$ and $\mathrm{CO}$ in AH Sco, UY Sct, and KW Sgr. The presence of molecular layers in the extended atmospheres of red supergiants is consistent with other observations of RSGs, such as VY CMa, $\mu$ Cep, and Betelgeuse (Perrin et al. 2004, 2005, Tsuji 2006; Ohnaka et al.2009, 2011, Wittkowski et al.2012).

In Figs. 113(3rd panels), we also show the uniform disk diameter predicted by the best-fit PHOENIX models. According to these results, the PHOENIX model is significantly more compact than observed. In particular, the observed extended water bands are not seen in the PHOENIX model predictions, and the observed CO layers are included in the models but significantly more compact compared to our observations. These findings are consistent with the observations of AGB stars by Marti Vidal et al. (2011).

The closure phases (bottom panels) show low values of $\leq 10$ deg in both bands $(\mathrm{K}-2.1 \mu \mathrm{m}$ and $\mathrm{K}-2.3 \mu \mathrm{m})$ and do not indicate deviations from point symmetry. However, because of the limited angular resolution of our observations that correspond to visibility measurements within the first lobe, we cannot exclude asymmetries on scales smaller than the stellar disk as observed, for instance, by Wittkowski et al. (2012) for VY CMa. 


\section{A new grid of PHOENIX model atmospheres}

After the data reduction, we compared our results to PHOENIX model atmosphere predictions to determine the angular diameter of our sources and their fundamental parameters. Here, we describe the general properties of our new grid of PHOENIX model atmospheres, while the comparison to our data is presented below in Sects. 4 and 5. We used version 16.03 of the PHOENIX code (for a general description see Hauschildt \& Baron 1999). The code assumes a hydrostatic atmosphere and local thermodynamic equilibrium (LTE) and computes $7 \cdot 10^{5}$ atomic lines and $9 \cdot 10^{7}$ molecular lines. Comparing results obtained with a spherical geometry and a plane-parallel geometry, the difference between the two geometries is greater for lower $\mathrm{T}_{\mathrm{eff}}$ (Hauschildt et al. 1999). As our targets are expected to show relatively low effective temperatures between $3200 \mathrm{~K}$ and $3700 \mathrm{~K}$, we used a spherical geometry.

We computed a new grid of a total of 127 PHOENIX model atmospheres with effective temperatures ranging between $2500 \mathrm{~K}$ and $3900 \mathrm{~K}$, surface gravities between $\log (\mathrm{g})=-0.5$ and $\log (\mathrm{g})=4.0$ in steps of 0.5 , and masses of $1 \mathrm{M}_{\odot}$ and $20 \mathrm{M}_{\odot}$. We also computed models with a low mass because we plan to fit these models to red giants in a future work. We used solar metallicity and a micro-turbulent velocity of $2 \mathrm{~km} / \mathrm{s}$ for all models. We tabulated model intensity profiles at 64 viewing angles for wavelengths from $1.8 \mu \mathrm{m}$ to $2.5 \mu \mathrm{m}$ in steps of $0.01 \AA$. Afterward, we averaged the monochromatic intensity profiles to match the spectral channels of the individual observations and computed the flux integrated over the stellar disk, as well as the visibility values for the baseline used (for more information on this procedure, see Wittkowski et al. 2003).

The modeling of atmospheres of cold giant stars, such as AH Sco, UY Sct, and KW Sgr, is complicated because of two effects, the treatment of molecular opacities and their spherical extension of the atmospheres (Hauschildt et al.1999). In the next section we discuss the comparison to our data and the impact of these effects.

\section{Fundamental parameters}

We estimated fundamental stellar properties of our sources, in particular the effective temperature and luminosity, to place them on the HR diagram and to compare them to stellar evolutionary tracks. The estimate of the effective temperature was based on our interferometric measurement of the angular diameter and the bolometric flux. The luminosity was derived based on the bolometric flux and the adopted distance.

Previous observations of RSGs have shown that continuum interferometric data near wavelengths of $2.20 \mu \mathrm{m}$ are represented well by simple limb-darkened disks as predicted by hydrostatic model atmospheres (Perrin et al.2004, Ohnaka et al.2009, 2011. Wittkowski et al. 2012). Haubois et al. (2009) obtained similar results in the $H$-band. Here, we fitted our continuum data at wavelengths of $2.15-2.25 \mu \mathrm{m}$ to our new PHOENIX model atmospheres and derived the angular diameter corresponding to the model layer where the Rosseland optical depth equals $2 / 3$ (the "Rosseland angular diameter"). We used a scaled visibility function of the form

$V\left(A, \theta_{\mathrm{Ross}}\right)=A * V^{\mathrm{PHOENIX}}\left(\theta_{\mathrm{Ross}}\right)$,

where $V^{\text {PHOENIX }}\left(\theta_{\text {Ross }}\right)$ is the synthetic visibility function based on the PHOENIX model with Rosseland angular diameter $\theta_{\text {Ross }}$ ) alone, and where $A$ allows the attribution of a fraction of the flux to an over-resolved dust component.
We used initial parameters of the PHOENIX model based on the effective temperature values by van Loon et al. (2005) of $\mathrm{T}_{\text {eff }}=3574 \mathrm{~K}$ for AH Sco and UY Sct and of $\mathrm{T}_{\mathrm{eff}}=3895 \mathrm{~K}$ for KW Sgr. We adopted a surface gravity of $\log (\mathrm{g})=-0.5$ for red supergiants. With these models, we obtained the Rosseland angular radius. Then, we derived the fundamental parameters using the Rosseland angular radius, the bolometric flux, and the distance (as described below in this section). After that, we derived the surface gravity $\log (\mathrm{g})$ from the linear Rosseland radius and the assumed stellar mass (estimated from the position of the stars in the HR diagram, 7-10 $\mathrm{M}_{\odot}$ ). Then, we obtained a new model using the effective temperature and $\log (\mathrm{g})$ of our grid closest to the derived values (keeping the chemical composition, micro-turbulent velocity, and stellar mass fixed). We iterated it until the effective temperature and $\log (\mathrm{g})$ converged. Finally, we used PHOENIX model atmospheres with parameters $T_{\text {eff }}=3600 \mathrm{~K}, \log (g)=-0.5$ for AH Sco; $T_{\text {eff }}=3400 \mathrm{~K}$, $\log (g)=-0.5$ for UY Sct; and $T_{\text {eff }}=3700 \mathrm{~K}, \log (g)=0.0$ for KW Sgr. We chose $M=20 \mathrm{M}_{\odot}$. Our current model grid includes only masses of $1 \mathrm{M}_{\odot}$ and $20 \mathrm{M}_{\odot}$, since the structure of the atmospheres is not very sensitive to mass (Hauschildt et al.1999), and the differences in our final parameters based on $1 \mathrm{M}_{\odot}$ and $20 \mathrm{M}_{\odot}$ models are well within the errors.

We obtained best-fit Rosseland angular diameter values of $\theta_{\text {Ross }}=(5.81 \pm 0.15) \mathrm{mas},(5.48 \pm 0.10) \mathrm{mas}$, and $(3.91 \pm 0.25)$ mas for AH Sco, UY Sct, and KW Sgr, respectively, together with scale factors $A$ of $0.81,0.94$, and 1.0. For KW Sgr, we initially obtained a scale factor of 1.08 , which we reset to 1.0 , since a factor greater than unity is not physically possible owing to a dust component. Using a scaled UD model instead of the PHOENIX model atmospheres, we obtained UD angular diameter values of $\theta_{\mathrm{UD}}=(5.64 \pm 0.15)$ mas for AH Sco, $(5.32 \pm 0.10)$ mas for UY Sct, and $(3.82 \pm 0.25)$ mas for KW Sgr. The errors include the statistical errors and systematic errors caused by calibration uncertainties. We estimated these errors from the differences between the visibility curves lying at the maximum and minimum of our data.

Figure 4 shows the average of continuum visibility data as a function of spatial frequency (one average per baseline and band). Here, the drawn error of the visibility data was computed as an average of the individual errors, since the errors are dominated by systematic effects. However, the model fit was based directly on the individual data points. Also shown are the maximum and minimum curves corresponding to our adopted error of the angular diameter. The synthetic flux and visibility values of the best-fit models are indicated in Figs. 1, 2, and 3, together with the observed values and with the best-fit UD model, in all wavelengths.

To obtain the bolometric flux of our targets, we used $U R I$ magnitudes from Humphreys \& Ney (1974), BV magnitudes from Kharchenko (2001), $J H K$ magnitudes from Cutri et al (2003), and the IRAS fluxes (IRAS 1986). We converted the magnitudes into fluxes using the zero values from the Skinner (1996) and the 2MASS (Cohen et al.2003) system.

We dereddened the flux values using an estimated $E_{B-V}$ value based on the $V-K$ color excess method. KW Sgr belongs to the OB5 Sgr association. Here, we used the B stars of this association to calculate a mean $E_{B-V}$ value. AH Sco and UY Sct are not known to belong to a cluster, so that we estimated $E_{B-V}$ using their own magnitudes. We used the ratios $A_{K} / A_{V}=0.12$, $A_{V} / E_{B-V}=3.69$ for the $\mathrm{M}$ stars and $A_{V} / E_{B-V}=3.1$ for the B stars from Fiorucci \& Munari (2003). We estimated the dereddened value $\left(A_{\lambda}\right)$ using $A_{\lambda}=\left(\alpha_{\lambda}+\beta_{\lambda} * E_{B-V}\right) * E_{B-V}$, where $\alpha_{\lambda}$ and $\beta_{\lambda}$ are taken for each filter from Morol (2000). We obtained 
Table 2. Photometry datasheet

\begin{tabular}{lccccccccccccc}
\hline \hline AH Sco & $\mathrm{U}$ & $\mathrm{B}$ & $\mathrm{V}$ & $\mathrm{R}$ & $\mathrm{I}$ & $\mathrm{J}$ & $\mathrm{H}$ & $\mathrm{Ks}$ & $\mathrm{L}$ & 12 & 25 & 60 & 100 \\
$\lambda(\mu \mathrm{m})$ & 0.36 & 0.45 & 0.55 & 0.7 & 0.9 & 1.235 & 1.662 & 2.159 & 2.4 & - & - & - & - \\
$\operatorname{mag}$ & - & 10.03 & 7.46 & 5.37 & 2.55 & 1.88 & 1.008 & 0.415 & - & - & - & - \\
Flux $\left(10^{-11} \mathrm{~W} / \mathrm{m}^{2} \mu \mathrm{m}\right)$ & - & 0.65 & 7.42 & 12.5 & 79.6 & 55.02 & 44.8 & 29.2 & - & 1.31 & 0.168 & 0.006 & 0.001 \\
\hline UY Sct & $\mathrm{U}$ & $\mathrm{B}$ & $\mathrm{V}$ & $\mathrm{R}$ & $\mathrm{I}$ & $\mathrm{J}$ & $\mathrm{H}$ & $\mathrm{Ks}$ & $\mathrm{L}$ & 12 & 25 & 60 & 100 \\
$\lambda(\mu \mathrm{m})$ & 0.36 & 0.45 & 0.55 & 0.7 & 0.9 & 1.235 & 1.662 & 2.159 & 2.4 & - & - & - & - \\
$\operatorname{mag}$ & 14.89 & 11.6 & 9.00 & 6.60 & 4.04 & 2.34 & 1.24 & 0.73 & 0.28 & - & - & - & - \\
Flux $\left(10^{-11} \mathrm{~W} / \mathrm{m}^{2} \mu \mathrm{m}\right.$ & 0.0046 & 0.14 & 0.88 & 4.03 & 20.5 & 36.1 & 36.2 & 21.9 & 6.26 & 0.54 & 0.093 & 0.003 & 0.008 \\
\hline KW Sgr & $\mathrm{U}$ & $\mathrm{B}$ & $\mathrm{V}$ & $\mathrm{R}$ & $\mathrm{I}$ & $\mathrm{J}$ & $\mathrm{H}$ & $\mathrm{Ks}$ & $\mathrm{L}$ & 12 & 25 & 60 & 100 \\
$\lambda(\mu \mathrm{m})$ & 0.36 & 0.45 & 0.55 & 0.7 & 0.9 & 1.235 & 1.662 & 2.159 & 2.4 & - & - & - & - \\
$\operatorname{mag}$ & 14.66 & 11.45 & 8.98 & 6.40 & 3.82 & 2.83 & 1.77 & 1.27 & 0.91 & - & - & - & - \\
Flux $\left(10^{-11} \mathrm{~W} / \mathrm{m}^{2} \mu \mathrm{m}\right.$ & 0.0057 & 0.18 & 0.89 & 4.85 & 24.7 & 22.98 & 22.15 & 13.27 & 3.50 & 0.52 & 0.07 & 0.0015 & 0.009 \\
\hline
\end{tabular}

Notes. The broadband photometry was obtained from Humphreys \& Ney (1974), Kharchenko (2001), Cutri et al (2003), and IRAS (1986). Zeromagnitude flux values are from Skinner (1996) for $U B V R I$ filter and from Cohen et al. (2003) for $J H K$ filter. The flux values given in this table are not dereddened.

$E_{B-V}$ values of $0.54 \mathrm{mag}$ for AH Sco, $0.92 \mathrm{mag}$ for UY Sct, and 0.98 mag for KW Sgr. For comparison, Levesque et al. (2005) estimate $E_{B-V}=0.93 \pm 0.33$ for KW Sgr. Finally, we integrated the spectro-photometric values to obtain the bolometric flux.

It is difficult to find information on the variability of the bolometric flux in RSGs. We (conservatively) estimated the variability of the bolometric flux using an extrapolation of the relationship obtained by Whitelock et al. (2000) related to the Hipparcos variability, although this relationship was derived from AGB stars, mostly Mira stars, with larger variability. The final estimated errors are of $15 \%$ for UY Sct and KW Sgr, and of $20 \%$ for AH Sco. The effective temperature was estimated based on the Rosseland angular diameter and the bolometric flux.

The adopted distances to our targets are taken from Melnik \& Dambis (2009) for KW Sgr and from Chen \& Shen (2008) for AH Sco. For UY Sct we used the mean of the distances obtained by Sylvester et al. (1998) and the distance obtained with the rule of Jura et al. (1989, $\mathrm{A}_{K} \sim 0.15$ per kpc distance).

Table 3 summarizes the fundamental parameters for our targets. The resulting effective temperatures are $\mathrm{T}_{\mathrm{eff}}=3682 \pm 190 \mathrm{~K}$ for AH Sco, $3365 \pm 134 \mathrm{~K}$ for UY Sct, and $3720 \pm 183 \mathrm{~K}$ for KW Sgr. Figure 5 shows the effective temperatures of our targets versus their spectral types as listed in Table 1 Also shown are three additional RSGs that have been observed in a similar way using the VLTI/AMBER instrument, which are VX Sgr (Chiavassa et al. 2010), Betelgeuse (Ohnaka et al. 2009, 2011), and VY CMa (Wittkowski et al.2012). We calculated the effective temperature and luminosity of Betelgeuse using the angular diameter obtained by Ohnaka et al. $\left(\theta_{\mathrm{LD}}=42.49 \pm 0.06 \mathrm{mas}\right)$, the distance from Harper et al. (2008) $(\mathrm{d}=197 \pm 47 \mathrm{pc})$, and the bolometric flux calculated by us in the same way as for our program stars $\left(f_{\text {bol }}=(1.07 \pm 0.16) 10^{-10} \mathrm{~W} \mathrm{~m}^{2}\right)$. We estimated the effective temperature to be $3620 \pm 137 \mathrm{~K}$. This value is consistent with the estimated by Perrin et al. (2004) of $3641 \pm 53 \mathrm{~K}$,

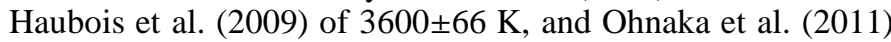
of $3690 \pm 54 \mathrm{~K}$. Likewise, we calculated the properties of VX Sgr using the photospheric angular diameter of $8.82 \pm 0.5$ mas by Chiavassa et al. (2010), the distance of $1570 \pm 270 \mathrm{pc}$ by Chen et al. (2007), and the bolometric flux calculated by us $\left(f_{\text {bol }}=(5.00 \pm 1.0) 10^{-9} \mathrm{~W} \mathrm{~m}^{2}\right)$.

It has been discussed that VX Sgr may be either a relatively massive AGB star or a red supergiant (Chiavassa et al. 2010). Our values suggest that VX Sgr is an RSG, so we used the spectral type of M4 Ia by Humphreys et al. (1972) instead of the Simbad classification of M5/M6 III by Houk \& Smith-Moore
Table 3. Fundamental parameters of AH Sco, UY Sct, and KW Sgr

\begin{tabular}{lcccc}
\hline \hline Parameter & AH Sco & UY Sct & KW Sgr & Ref. \\
\hline $\mathrm{f}_{\text {bol }}$ & $2.07 \pm 0.41$ & $1.28 \pm 0.19$ & $0.97 \pm 0.15$ & 1 \\
$\left(10^{-9} \mathrm{~W} \mathrm{~m}^{-2}\right)$ & & & & \\
$\mathrm{d}(\mathrm{pc})$ & $2260 \pm 190$ & $2900 \pm 317$ & $2400 \pm 300$ & 2 \\
$\mathrm{~L}\left(10^{32} \mathrm{~W}\right)$ & $1.26 \pm 0.33$ & $1.29 \pm 0.34$ & $0.67 \pm 0.20$ & 3 \\
$\log \left(\mathrm{L} / \mathrm{L}_{\odot}\right)$ & $5.52 \pm 0.26$ & $5.53 \pm 0.27$ & $5.24 \pm 0.29$ & - \\
$\theta_{\text {Ross }}(\mathrm{mas})$ & $5.81 \pm 0.15$ & $5.48 \pm 0.10$ & $3.91 \pm 0.25$ & 4 \\
$\left.\mathrm{R}_{(} \mathrm{R}_{\odot}\right)$ & $1411 \pm 124$ & $1708 \pm 192$ & $1009 \pm 142$ & 5 \\
$\mathrm{~T}_{\text {eff }}(\mathrm{K})$ & $3682 \pm 190$ & $3365 \pm 134$ & $3720 \pm 183$ & 6 \\
$\log \left(\mathrm{T}_{\text {eff }}\right)$ & $3.57 \pm 0.05$ & $3.53 \pm 0.04$ & $3.57 \pm 0.05$ & - \\
\hline
\end{tabular}

Notes. 1: Humphreys \& Ney (1974), Kharchenko (2001), Cutri et al (2003), IRAS (1986). 2: Chen \& Shen 2008 (AH Sco), Sylvester et al. 1998 (UY Sct), Melnik, A. M., Dambis, A. K.,2009. (KW Sgr). 3: from 2 and 1; 4: This work; 5: from 4 and 2; 6: from 5 and 3.

(1988). For comparison, Fig. 5 includes the calibrations of the effective temperature scales by Humphreys \& McEloy (1984), Massey \& Olsen (2003), Levesque et al. (2005), and van Belle et al. (2009). Our values confirm the more recent calibrations by Levesque et al. and van Belle et al. In particular, KW Sgr and Betelgeuse are in very good agreement with the scale by Levesque et al., while the cooler sources show a larger scatter, but are consistent with both of these calibrations within 1-2 $\sigma$. This scatter may be caused by an erroneous calibration, and also conceivable variations of the spectral type (Humphreys et al. 1972).

Figure 6 shows the positions of AH Sco, UY Sct, and KW Sgr in the Hertzsprung-Russell (HR) diagram, together with the recent evolutionary tracks from Ekström et al. (2012). Also shown are the positions of VY CMa, Betelgeuse, and VX Sgr as discussed above. The targets are located close to the red limits of these tracks. AH Sco and UY Sct are close to the evolutionary tracks corresponding to an initial mass of $25 \mathrm{M}_{\odot}$ with rotation or 30-40 $\mathrm{M}_{\odot}$ without rotation. KW Sgr is consistent with evolutionary tracks corresponding to masses ranging from $20 \mathrm{M}_{\odot}$ with or without rotation, to $40 \mathrm{M}_{\odot}$ without rotation. The positions of our targets with higher mass $\left(\geq 20 \mathrm{M}_{\odot}\right)$ are drawn slightly to the right of the Hayashi limit. This may be consistent with Fig. 3c of Levesque et al. (2005), where several sources of this mass are placed slightly to the right, too. This may indicate that sources of this mass are not in hydrostatic equilibrium. Our measurements are based on direct measurements of the angular diam- 

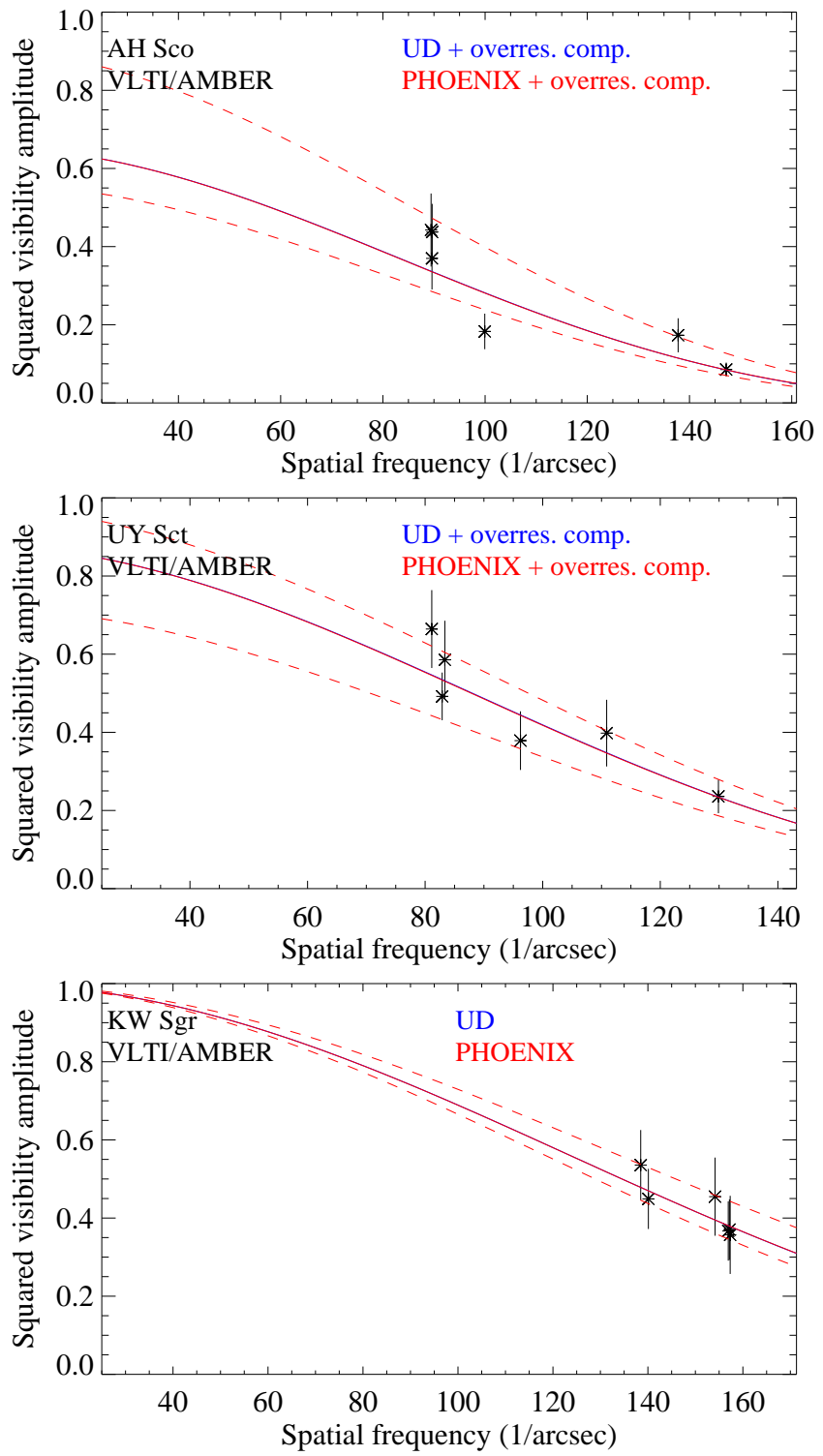

Fig. 4. Average of squared visibility amplitudes taken in the near-continuum bandpass at $2.15-2.25 \mu \mathrm{m}$ (one average per baseline and band) for AH Sco (top), UY Sct (middle), and KW Sgr (bottom) as a function of spatial frequency. The red lines indicate the best-fit UD models and the blue lines the bestfit PHOENIX models. The dashed lines are the maximum and minimum visibility curves, from which we estimated the errors of the angular diameters.

eter and the bolometric flux, and thus provide an independent determination of the location of red supergiants in the HR diagram compared to the approach by Levesque et al. (2005), which was based on a comparison of spectro-photometry to hydrostatic MARCS model atmospheres.

\section{Atmospheric structure}

Previous studies have demonstrated that near-infrared spectra of RSGs are predicted reasonably well by static PHOENIX model atmospheres (Lançon et al. 2007), but that discrepancies are observed in detailed spectral features toward the coolest stars. Lançon et al. noted that these discrepancies may be related to

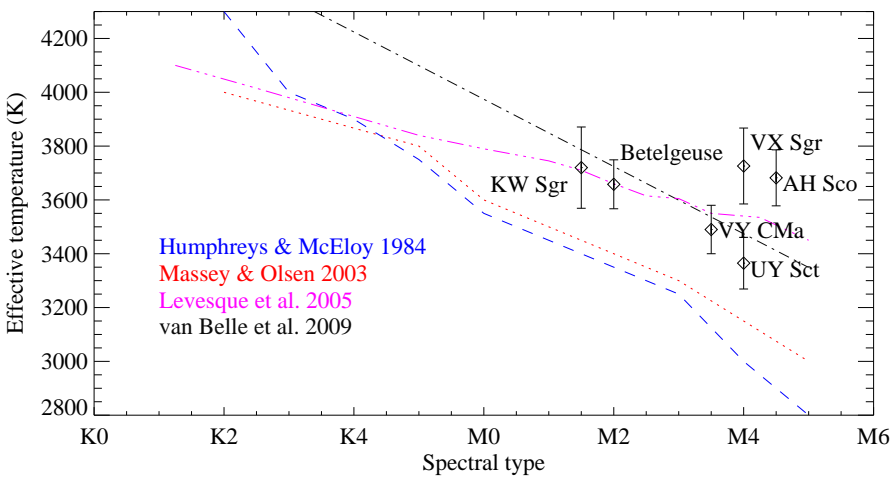

Fig. 5. Effective temperature versus spectral type of our sources and VY CMa, VX Sgr and Betelgeuse. Also we show the scales of Humphreys \& McElroy (1984), Massey \& Olsen (2003), Levesque et al. (2005), and van Belle et al. (2009)

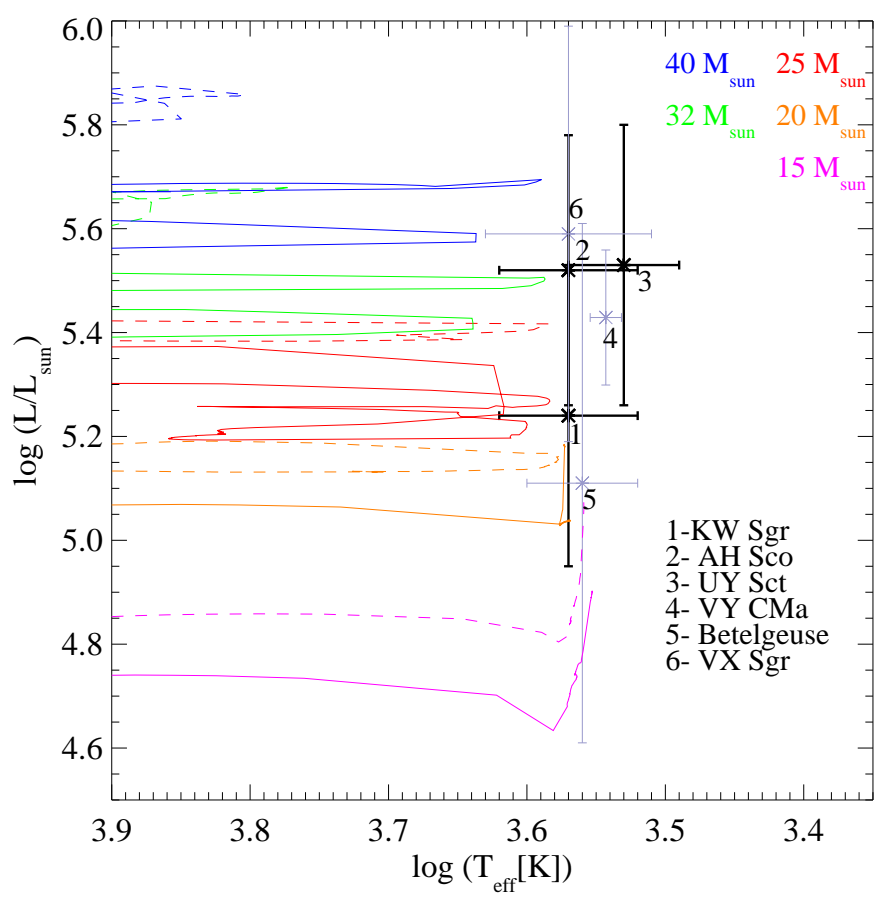

Fig. 6. Location of AH Sco, UY Sct, and KW Sgr in the HR diagram using our fundamental parameters. Also shown is the position of VY CMa from Wittkowski et al. (2012), Betelgeuse from Ohnaka et al. (2011), and VX Sgr from (Chen et al. 2007 and Chiavassa et al. (2010). The positions of the stars are comopared to evolutionary tracks from Ekström et al. (2012) for masses of $15 \mathrm{M}_{\odot}, 20 \mathrm{M}_{\odot}, 25 \mathrm{M}_{\odot}, 32 \mathrm{M}_{\odot}$, and $40 \mathrm{M}_{\odot}$. The solid lines are models without rotation, and the dashed lines are with rotation.

the variability of these sources and the higher micro-turbulent velocities.

The top panels of Figs. 1,3 indicate the flux spectra of the best-fit PHOENIX model atmospheres compared to the observed flux spectra. They show a good agreement of the overall shapes of the $1.9-2.5 \mu \mathrm{m}$ spectra, and in particular good agreement of the strengths of the $\mathrm{CO}$ bandheads. The largest deviations are observed for the spectral shape of the very cool M4 supergiant UY Sct. Overall, our results on the $K$-band spectra are consistent with those by Lançon et al. (2007). 
The synthetic interferometric visibility spectra of the bestfit PHOENIX models are compared to the observed visibility spectra in the second panels of Figs. 1,3, Considering the errors bars, the continuum visibility values are consistent with a limb-darkened disk. This comparison shows that the large observed drops of the visibility function at the locations of the $\mathrm{CO}$ bandheads between $2.3 \mu \mathrm{m}$ and $2.5 \mu \mathrm{m}$, as well as of the broad $\mathrm{H}_{2} \mathrm{O}$ feature centered on $1.9 \mu \mathrm{m}$ are not reproduced by the PHOENIX model atmospheres that we used here. The synthetic visibility spectra show features at the location of the $\mathrm{CO}$ bandheads, but which are much weaker than the observed features. The observed drops of the visibility at the $\mathrm{H}_{2} \mathrm{O}$ feature centered on $1.9 \mu \mathrm{m}$ are not visible in the model visibility spectra.

These results indicate that the opacities of $\mathrm{CO}$ are included in the PHOENIX models providing a satisfactory fit to the $K$-band spectra, but that the extension of these molecular layers are too compact in the models compared to our observations. This result is consistent with the similar observations of the RSG VY CMa by Wittkowski et al. (2012) and the results shown by Lançon et al. (2007). The satisfactory fit to stellar spectra, together with an unsatisfactory fit to interferometric visibilities, illustrates the importance of interferometric observations in addition to spectral observations to fully constrain and test stellar model atmospheres, as noted previously by, e.g., Wittkowski et al. (2006) and Paladini et al. (2011). In a future work, it may be possible to extend PHOENIX model atmospheres farther out, for instance by attaching a slow wind to the atmosphere, or by considering NLTE in the molecular lines or 3D structures.

The levitation of gas to radii where dust particles can condense and be accelerated by radiative pressure, as observed here, is an important ingredient in our understanding of the formation of stellar winds of cool giant and supergiant stars (e.g. Bladh \& Höfner 2012). It is not yet clear which physical processes may levitate the gas in the case of supergiant stars. In the case of asymptotic giant branch (AGB) stars, it has been established that pulsation-induced shock waves lead to very extended atmospheres that reach to radii of a few photospheric radii (Jeong et al. 2003; Ireland et al. 2004, 2008, 2011). Recent interferometric observations of Mira variable AGB stars obtained with the AMBER instrument by Wittkowski et al. (2011) have shown extended molecular layers that are consistent with predictions by the latest hydrodynamical model atmospheres of Mira variables (Ireland et al.2011).

It is remarkable that the wavelength dependence of the visibility spectra observed here for RSGs is qualitatively similar to observations of AGB stars (Marti-Vidal et al. 2011; RuizVelasco et al. 2011; Wittkowski et al.2011) and to predictions by the latest hydrodynamic model atmospheres of Miras. This has also already been observed for VX Sgr (Chiavassa et al. 2010). Although the pulsation amplitudes of RSGs are generally lower than those of Mira variable AGB stars, we speculate that the same process of pulsation-induced shock waves may also lead to the extended molecular layers that we observed here for the RSGs AH Sco, UY Sct, and KW Sgr, and that have also been observed previously for other RSGs. Samus et al. (2009) list Vband amplitudes of 2-4 mag and pulsation periods around 700 days for AH Sco, KW Sgr, and UY Sct, compared to 4-6 mag and periods between 300-450 days for the Mira variables discussed in Wittkowski et al. (2011). Indeed, Heger et al. (1997) found that RSGs can have similar pulsation properties as AGB stars, based on their theoretical calculation. Dynamic model atmospheres based on stellar pulsation are not currently available for the parameter space of RSGs, so that this hypothesis cannot be tested within this paper. We encourage the development of such dynamic model atmospheres for RSGs.

$\mathrm{SiO}$ maser emission has been observed to form in ring-like structures around a large number of oxygen-rich evolved stars (cf., Benson et al. 1990). In the past, radii of the $\mathrm{SiO}$ maser emission were compared to stellar radii to obtain an additional constraint on the structure of the extended atmosphere and on the maser pumping mechanism. Most of these comparisons have been obtained for Mira variable stars. When meaningful stellar radii such as continuum radii or Rosseland radii were used and were obtained at similar phases as the $\mathrm{SiO}$ maps, the radii of the maser emission was consistently found close to two stellar radii (e.g., Boboltz \& Wittkowski 2005; Fedele et al. 2005; Cotton et al. 2010). This location is consistent with theoretical modeling by Humphreys et al. (1997) and Gray et al. (2009), and is predicted to be located close to the position of a shock front. Among the RSGs observed here, AH Sco is also a known source of $\mathrm{SiO}$ maser emission with a mean diameter of 17.2 mas (Chen $\&$ Shen 2008). Compared to our Rosseland angular diameter of 6.0 mas, the maser emission lies at 2.9 stellar radii. Similarly, the maser radius of VX Sgr of 13 mas by Su et al. (2012) lies at 3.0 stellar radii compared to the Rosseland angular diameter of 8.8 mas by Chiavassa et al. (2010). This might indicate that $\mathrm{SiO}$ maser of red supergiants are located at systematically larger distances from the stellar surface compared to AGB stars. Within a framework of stellar pulsation, this may be related to the larger pulsation periods and a different shock front behavior.

Convection has also been discussed as a possible process to dredge material up to higher layers and to produce surface inhomogeneities and shock waves in the photosphere (e.g., Chiavassa et al. 2011). Available 3D convection simulations for RSGs have been compared to interferometric data of VX Sgr and have not shown the typical observed wavelength dependence of the visibility function at the positions of the water band centered at $1.9 \mu \mathrm{m}$ and of the $\mathrm{CO}$ bandheads at $2.3 \mu \mathrm{m}-2.5 \mu \mathrm{m}$ (Chiavassa et al. 2010). Ohnaka et al. (2011) discuss that the observed time variation of the continuum data of the RSG Betelgeuse is much less than the maximum variation predicted by current $3 \mathrm{D}$ convection simulations, suggesting that the effect of photospheric convection may be overestimated in current models. Chiavassa et al. (2011) mention that with current models it is not clear whether convective motion is strong enough to explain the motion detected at extended layers up to 1.4 stellar radii, where the convective flux is low. While these results suggest that the effects of convection may be limited to the photospheric layers to a smaller degree than present in current models and may not reach into extended molecular layers, a detailed comparison of interferometric data to latest $3 \mathrm{D}$ convection simulations is required for a definite answer, and will be a part of our future work.

\section{Conclusions}

We obtained near-infrared spectro-interferometric observations of the RSGs AH Sco, UY Sct, and KW Sgr. Our data indicate the presence of extended molecular layers of $\mathrm{CO}$ and $\mathrm{H}_{2} \mathrm{O}$. We compared the spectra and visibilities to a new grid of hydrostatic PHOENIX model atmospheres.

Our near-infrared spectra of AH Sco, UY Sct, and KW Sgr are reproduced well by the PHOENIX model atmospheres; however, the synthetic visibility amplitudes of the model do not predict the large extensions of the molecular bands. This may be due to processes including pulsation or convection, which are not included in our currently available hydrostatic model atmo- 
spheres. These processes may explain the observed extensions of the atmospheres and the wind acceleration in RSGs.

The continuum $(2.15-2.25 \mu \mathrm{m})$ appears to be largely free of contamination by molecular layers. Thus, the PHOENIX model fitting to the continuum allows us to estimate meaningful stellar radii. Using the PHOENIX model in the continuum, we estimate a Rosseland diameter of $5.81 \pm 0.15$ mas $\left(R=1411 \pm 124 \mathrm{R}_{\odot}\right)$ for AH Sco, $5.48 \pm 0.10$ mas $\left(R=1708 \pm 192 \mathrm{R}_{\odot}\right)$ for UY Sct, and $3.91 \pm 0.25$ mas $\left(R=1009 \pm 142 \mathrm{R}_{\odot}\right)$ for $\mathrm{KW} \mathrm{Sgr}$. We also estimate effective temperatures of $3682 \pm 190 \mathrm{~K}$ for AH Sco, $3365 \pm 134 \mathrm{~K}$ for UY Sct, and $3720 \pm 183 \mathrm{~K}$ for KW Sgr.

With the effective temperature and the luminosity calculated from the Rosseland radius, the bolometric flux, and the distance, we locate our targets in the HR diagram. The positions of the stars in the HR diagram fall close to the red limit of the tracks corresponding to stars of mass around 25/30-40 $\mathrm{M}_{\odot}$ (AH Sco and UY Sct) and 20/20-40 $\mathrm{M}_{\odot}$ (KW Sgr) with/without rotation.

Spectro-interferometric observations of RSGs with spatially resolved extended molecular layers are still scarce. In this work, we studied three sources, which almost doubles the sample size.

Acknowledgements. We gratefully thank Juan Fabregat and Gerard van Belle for their great help with the calculation of the extinction values and the bolometric fluxes. This research made use of the AMBER data reduction package of the Jean-Marie Mariotti Center, and the SIMBAD database operated at the CDS, Strasbourg, France. B.A.T and J.M.M acknowledge support by the Spanish Ministry of Science and Innovation though the grant AYA2009-13036-C02-02.

\section{References}

Benson, P. J., Little-Marenin, I. R. \& Woods, T. C., et al. 1990, ApJS, 74, 911 Bessel, M. S., Castelli F., \& Plez, B., 1998, A\&A, 333, 231

Bladh, S., \& Höfner, S. 2012, A\&A, 546, A76

Boboltz, D. A., \& Wittkowski, M. 2005, ApJ, 618, 953

Campins, H., Rieke, G. H., \& Lebofsky, M. J., 1985, AJ, 90, 896

Chelli, A., Utrera, O. H., \& Duvert, G. 2009, A\&A, 502, 705

Chen, X., Shen, Z.-Q., \& Xu, Y. 2007, Chinese J. Astron. Astrophys., 7, 531

Chen, X., \& Shen, Z.-Q., 2008, ApJ, 681, 1574

Chiavassa, A., Lacour, S., Millour, F., et al. 2010, A\&A, 511, A51

Chiavassa, A., Freytag, B., Masseron, T. et al, 2011, A\&A, 535, A22

Cohen, M., Wheaton, Wm. A., \& Megeath, S. T., 2003, AJ, 126, 1090

Cotton, W. D., Ragland, S., Pluzhnik, E. A., et al. 2010, ApJS, 188, 506

Cutri, R. M., Skrutskie, M. F., van Dyk, S., et al. 2003, "The IRSA 2MASS All-Sky Point Source Catalog, NASA/IPAC Infrared Science Archive.

Ekstrom, S., Georgy, C., Eggenberger, P., et al. 2012, A\&A, 537, 146

Fedele, D., Wittkowski, M., \& Paresce, F., et al. 2005, A\&A, 431, 1019

Fiorucci, M., \& Munari, U., 2003, A\&A, 401, 781

Harper, G. M., Brown, A., \& Guinan, E. F. 2008, AJ, 135, 1430

Haubois, X., Perrin, G., Lacour, S., et al. 2009, A\&A, 508, 923

Hauschildt, P. H., Allard, F., Ferguson, J., Baron, E., \& Alexander, D. R. 1999, ApJ, 525, 871

Hauschildt, P. H., \& Baron , E. 1999, J. Comp. Appl. Math, 109, 41

Heger, A., Jeannin, L., Langer, N., \& Basaffe, I, 1997, A\&A, 327, 224

Houk, N., \& Smith-Moore, M. 1988, Michigan Catalogue of Two-dimensional Spectral Types for the HD Stars. Volume 4

Humphreys, R. M., Strecker, D. W., \& Ney, E. P. 1972, ApJ, 172, 75

Humphreys, R. M., \& Ney, E. P. 1974, ApJ, 194, 623

Humphreys, R. M., \& McElroy, D. B. 1984, ApJ, 284, 565

Humphreys, E. M. L., Gray, M. D., Field, D., Yates, J. A., \& Bowen, G. 1997, Ap\&SS, 251, 215

IRAS Catalogue of Point Sources (V2.0, 1986)

Ireland, M. J., Scholz, M., \& Wood, P. R. 2004, MNRAS, 352, 318

Ireland, M. J., Scholz, M., \& Wood, P. R. 2008, MNRAS, 391, 1994

Ireland, M. J., Scholz, M., \& Wood, P. R. 2011, MNRAS, 418, 114

Jeong, K. S., Winters, J. M., Le Bertre, T., \& Sedlmayer, E. 2003, A\&A, 407, 191

Johnson, H. L., 1965, CoLPL, 3, 73.

Jura, M., Joyce, R., R., \& Kleinmann, S. G., 1989, ApJ, 336, 936

Jura, M., \& Kleinmann, S. G., 1990, ApJ, 769, 73

Gray, M. D., Wittkowski, M., et al. 2009, MNRAS, 397, 51

Kervella, P., Verhoelst, T., Ridgway, S. T., et al. 2009, A\&A, 504, 115

Kervella, P., Perrin, G., Chiavassa, A., et al. 2011, A\&A, 531, A117

Kharchenko, N. V., 2001, KFNT, 17, 409
Lafrasse, S., Mella, G., Bonneau, D., et al. 2010, Proc. SPIE, 7734, 410 Lançon, A., \& Wood, P. R. 2000, A\&AS, 146, 217

Lançon, A., Hauschildt, P. H., Ladjal, D. \& Mouhcine, M., 2007, A\&A, 468, 205 Levesque, E. M., Massey, P., Olsen, K. A. G., et al. 2005, ApJ, 628, 973

Lord, S. D. 1992, NASA technical memorandum 103957

Mauron, N., Josselin, E., 2011, A\&A, 526, A156

Martí-Vidal, I., Marcaide, J. M., Quirrenbach, A., et al. 2011, A\&A, 529, A115

Massey, P., \& Olsen, K. A. G. 2003, ApJ, 126, 2867.

Massey, P., Levesque, E. M., \& Plez, B., 2006, ApJ, 646, 1203.

Melnik, A. M, \& Dambis, A. K., 2009, MNRAS, 400,518

Morel, M., \& Magnenat, P., 1978, A\&AS, 34, 477

Moro1, D., \& Munari1, U. 2000, A\&A, 147, 361

Moshir M., Kopan G., Conrow T., et al., 1992, Infrared Processing and Analysis Center (IPAC), California Institute of Technology

Ohnaka, K., Hofmann, K. H., Benisty, M., et al., 2009, A\&A, 503, 183

Ohnaka, K., Weigelt, G., Millour, F., et al., 2011, A\&A, 529, 163

Paladini, C., van Belle, G. T., Aringer, B., et al. 2011, A\&A, 533, A27

Perrin, G., Ridgway, S. T., Coudé du Foresto, V., et al., 2004, A\&A, 418, 675.

Perrin, G., Ridgway, S. T., Verhoelst, T., et al. 2005, A\&A, 436, 317

Petrov, R. G., Malbet, F., Weigelt, G., et al. 2007, A\&A, 464,1

Tarafdar, \& S. P., 1988, ApJ, 331, 932

Tatulli, E., Millour, F., Chelli, A., et al. 2007, A\&A, 464, 29

Tsuji, T. 2006, ApJ, 645, 1448

Ruiz-Velasco, A. E., Wittkowski, M., Wachter, A., Schröder, K.-P., \& Driebe, T. 2011, A\&A, 535, A100

Samus, N. N., Durlevich, O. V., \& et al. 2009, VizieR Online Data Catalog, 2025

Skinner, C. J., 1996, Instrument Science Report NICMOS-014

Su, J. B., Shen, Z.-Q., \& Chen, X., et al. 2012, ApJ, 754, 47

Sylvester, R. J., Skinner, C. J., \& Barlow, M. J., MNRAS, 301, 1083

van Belle, G. T., Creech-Eakman, M. J., \& Hart, A., 2009, MNRAS, 394, 1925.

Van Loon, J. Th., Cioni, M. R. L., Zijlstra, A. A. et al. 2005, A\&A, 438, 273

Whitelock, P., Feast, M., 2000, MNRAS 319, 728

Wittkowski, M., Aufdenberg, J., P, \& Kervella, P. 2003, A\&A, 413, 711

Wittkowski, M., Aufdenberg, J. P., Driebe, T., et al. 2006, A\&A, 460, 855

Wittkowski, M., Boboltz, D. A., Ireland, M., et al. 2011, A\&A, 532L, 7

Wittkowski, M., Hauschildt, P. H., Arroyo-Torres, B., \& Marcaide, J. M., 2012, A\&A, 540, 12 

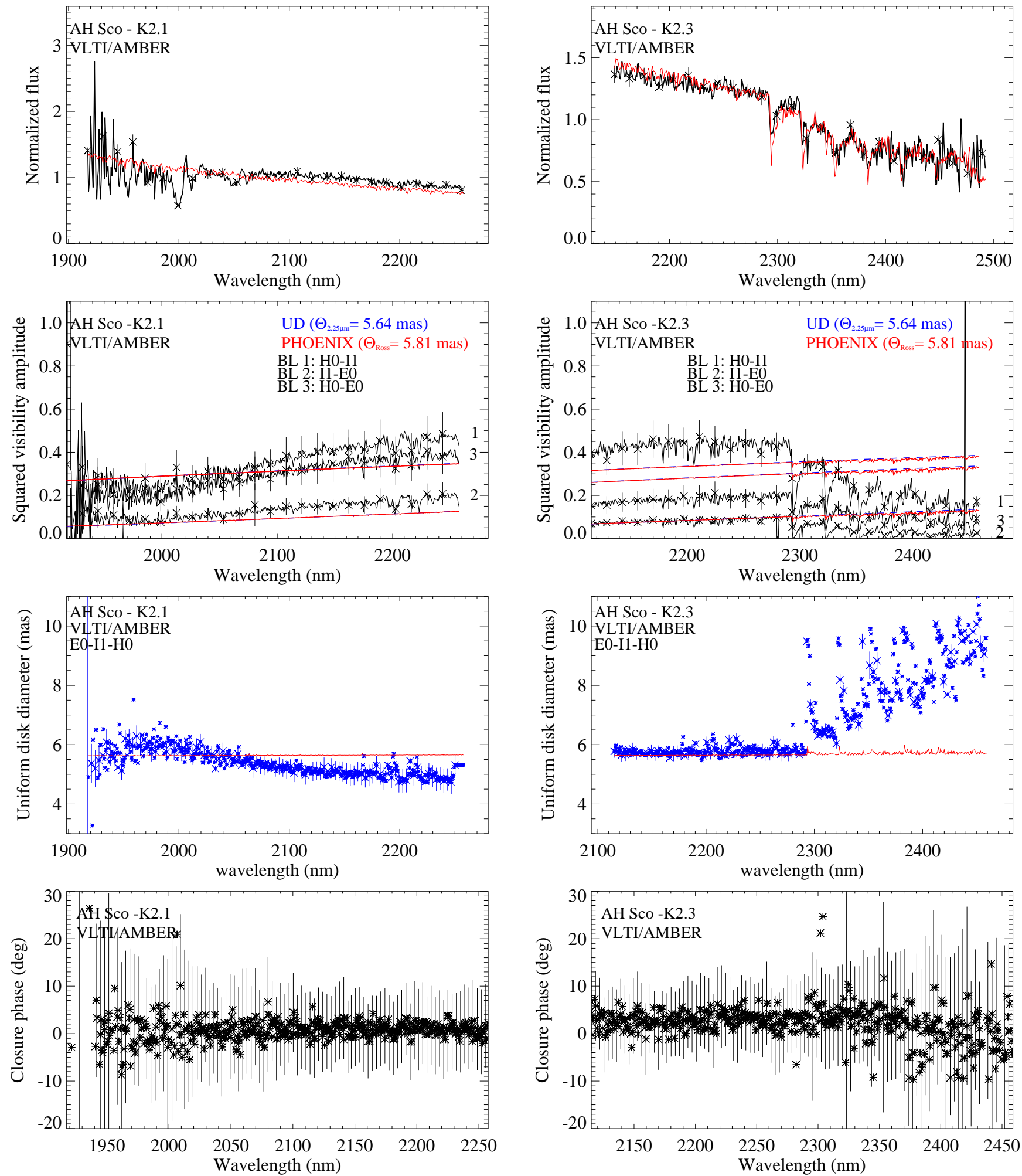

Fig. 1. Left: Observed (black) normalized flux, squared visibility amplitudes, UD diameters predicted form our data (blue) and from the best-fit PHOENIX model (red), and closure phases in degree (from top to bottom) of AH Sco obtained with the MR-K $2.1 \mu \mathrm{m}$ setting. Right: Same as left but obtained with the MR-K $2.3 \mu \mathrm{m}$ setting. The blue curves show the best-fit UD model, and the red curves the best-fit PHOENIX model prediction. For the baseline lengths and position angles, see Table 1 In the 2nd panel on the left, two model lines overlap, corresponding to baselines of $40.6 \mathrm{~m}$ and $40.4 \mathrm{~m}$. 

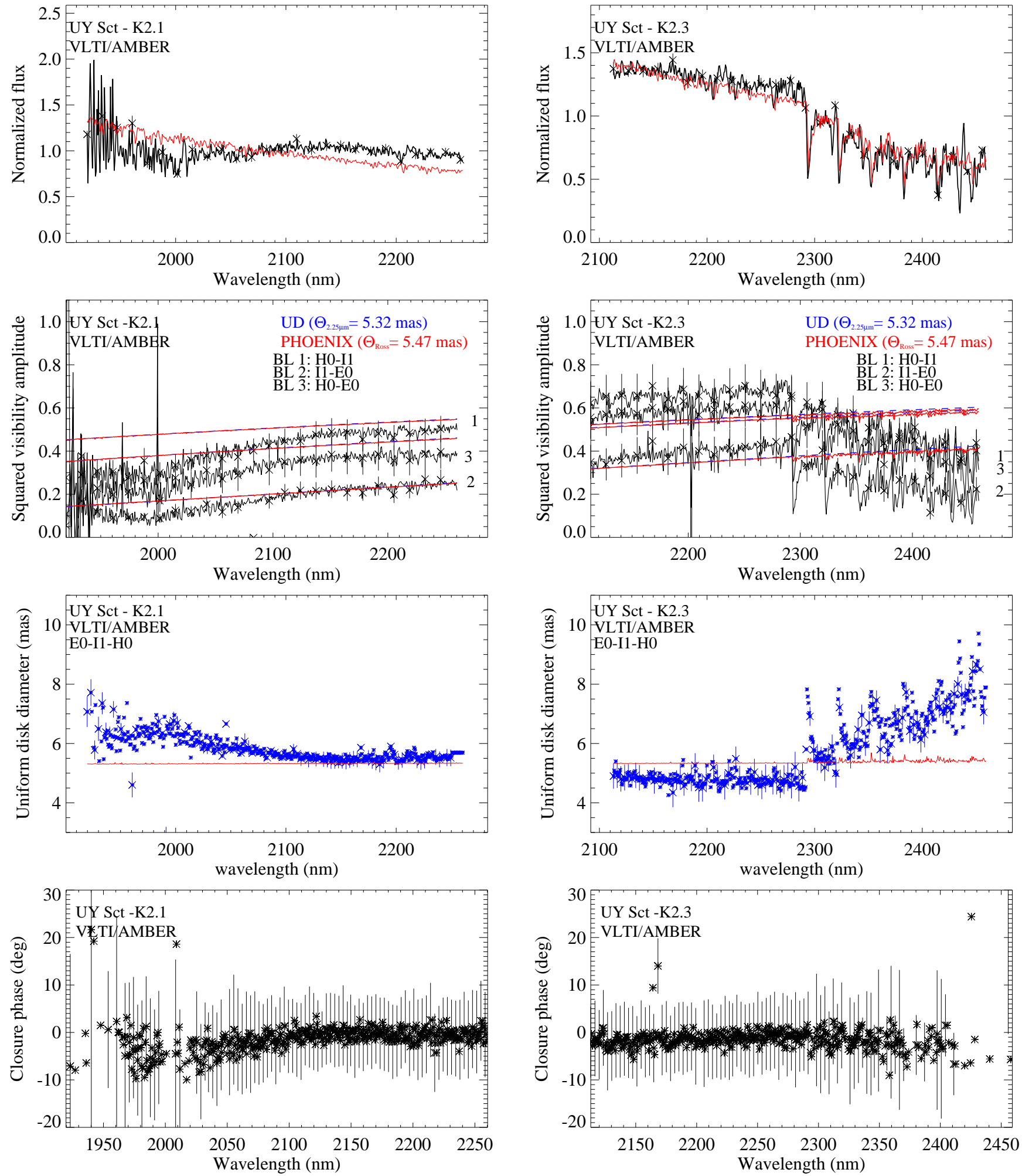

Fig. 2. As Fig. 1, but for UY Sct. 

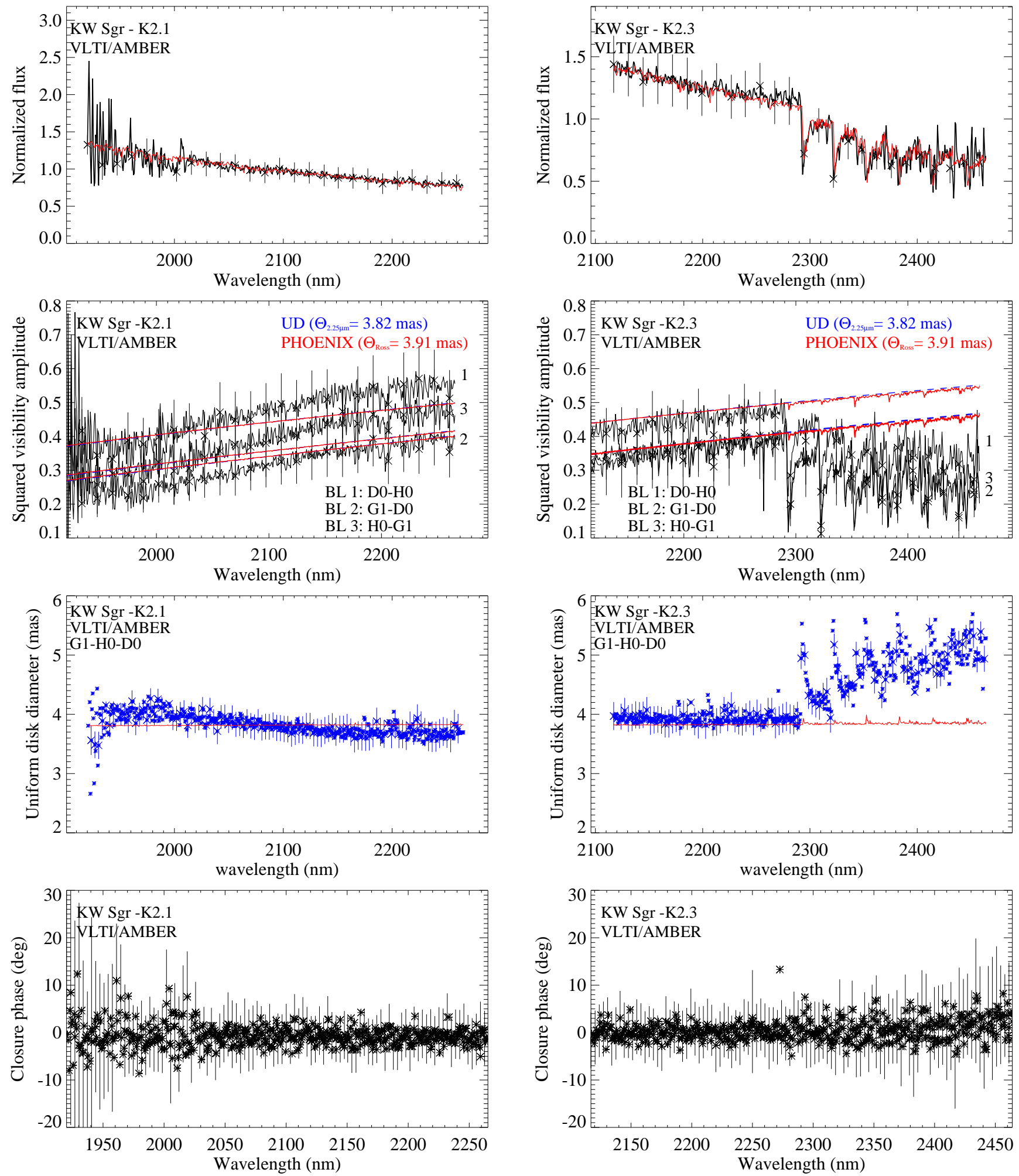

Fig. 3. As Fig. 1, but for KW Sgr. 